\title{
Evaluation sozialpolitischer Reformen
}

\author{
Patrick A. Puhani · Katja Sonderhof
}

Angenommen: 16. Februar 2011 / Online publiziert: 29. März 2011

(C) Institut für Arbeitsmarkt- und Berufsforschung 2011

Zusammenfassung Dieser Beitrag illustriert die Evaluation sozialpolitischer Regeländerungen anhand von zwei Reformen, die Eingriffe in die Rechte der Arbeitgeber und Arbeitnehmer vornahmen. Kausale Effekte der Reformen werden anhand von Kontrollgruppen-Ansätzen ermittelt (hier Differenz-von-Differenzen-Schätzungen). Die Ergebnisse zeigen, dass Eingriffe des Staates in den Arbeitsmarkt neben den gewollten positiven unterstützenden Effekten - im einen Fall für Kranke im anderen Fall für Eltern - auch negative Effekte haben. Die Verpflichtung des Arbeitgebers, einem abwesenden Arbeitnehmer 100 Prozent statt 80 Prozent des Lohnes zu bezahlen, erhöht die durchschnittlichen Abwesenheitstage vom Arbeitplatz von ca. 6 auf ca. 8 Tage (Abweichungen je nach Spezifikation; einige Schätzungen liefern sogar noch etwas größere Effekte), ohne das subjektive Gesundheitsempfinden der Arbeitnehmer nachweislich negativ zu beeinträchtigen. Die Verpflichtung des Arbeitgebers, Eltern bis zu 3 Jahre den Arbeitsplatz auch bei Abwesenheit zu garantieren, reduziert die Weiterbildung für junge Frauen, die der Arbeitgeber selbst anordnet.

JEL Klassifikationen I18 · J16 · J24 · J83

\section{Evaluation of social policy reforms}
Abstract This paper illustrates the evaluation of changes in social policy regulations with respect to two reforms that strengthened the rights of either employers or em-
P.A. Puhani $(\varangle) \cdot$ K. Sonderhof
Institut für Arbeitsökonomik, Leibniz Universität Hannover,
Königsworther Platz 1, 30167 Hannover, Deutschland
e-mail: puhani@aoek.uni-hannover.de
K. Sonderhof
e-mail: sonderhof@aoek.uni-hannover.de

ployees. Causal effects of the reform are based on control group designs (difference-in-differences estimates). The results show that interventions of the state in the labor market also have negative effects-besides the desired supportive/distributional effects for sick people in one case and for parents in the other. The regulation that the employer has to pay an absent sick worker 100 instead of 80 percent of the wage increases the average days of absence from work from about 6 to 8 days (estimates vary by specification, some estimates even exhibit higher effects), without significantly negatively affecting subjective health indicators. The regulation that demands employers to guarantee comparable employment to a parent even after 3 years of absence reduces employer-arranged training for young women.

Keywords Sick pay · Maternity leave $\cdot$ Health · Training

\section{Einleitung}

Die soziale Marktwirtschaft der Bundesrepublik Deutschland steht vor großen Herausforderungen. Anhaltende Reformbemühungen in den Bereichen Gesundheit, Alterssicherung, Lohnersatzleistungen und Familienpolitik verdeutlichen die Breite der Problematik. Geringe Geburtenzahlen, längere Lebensdauern sowie niedrige Beschäftigungsquoten gehören zu den wesentlichen Determinanten der Fragilität des Sozialstaates in seiner heutigen Form. Hinzu kommt, dass durch den demographischen Wandel der Anteil der Erwerbspersonen an der Bevölkerung in den kommenden Jahrzehnten abnehmen wird, was zu einer zusätzlichen Belastung des Sozialsystems führen wird. Bekenntnisse der politischen Entscheidungsträger zum System ,,soziale Marktwirtschaft" lassen häufig die Dynamik außer Acht, die der Sozialstaat in den letzten Jahrzehnten entwickelt hat. 
Seit den späten 1950er Jahren wurde der wirtschaftliche Erfolg des jungen deutschen Staates zunehmend auch für eine Intensivierung des sozialen Ausgleiches genutzt. Beispiele sind höhere Leistungen für Arbeitslose, ein verlängerter Erziehungsurlaub oder die schrittweise Abschaffung von Karenztagen. Neben der politisch gewünschten Verteilungswirkung entstanden durch die genannten Reformen auch Anreize bezüglich des Arbeitsangebots und der Arbeitsnachfrage, die jedoch in früheren Jahrzehnten (vermutlich aufgrund der guten wirtschaftlichen Lage und auch der mangelnden Verwertbarkeit von Personendaten) nicht erforscht wurden.

Ziel dieses Beitrags ist es zu illustrieren, wie auf Basis von Personendaten mittels ökonometrischer Analysen kausale Effekte sozialstaatlicher Reformen auf den Arbeitsmarkt evaluiert werden können. ${ }^{1}$ Nach dem Ende des zweiten Weltkrieges wurde der Sozialstaat in Deutschland sukzessive ausgebaut und erweitert. So wurde in verschiedenen Bereichen (Rentenversicherung, Krankenversicherung, Lohnersatzleistungen, Familienpolitik) ein Ausgleichs- und Umverteilungsmechanismus geschaffen. Diese Reformen hatten neben Umverteilungs- auch Anreizeffekte, die sich noch heute in vielerlei Hinsicht auf den Arbeitsmarkt auswirken. Einen Überblick über einen Großteil der sozialpolitischen Reformen geben Frerich und Frey (1993), Frerich (1996), Lampert und Althammer (2001), Lampert und Bossert (1992) oder auch Metzler (2003).

Empirische Evaluationen sind für viele Reformen der Sozialpolitik entweder selten oder sie fehlen gänzlich. Dies liegt auch daran, dass es für frühere Jahre kaum geeignete Daten gibt, um Änderungen in der Sozialpolitik mit Kontrollgruppenansätzen zu evaluieren. So wurde z.B. das sozio-ökonomische Panel (SOEP) des DIW in Berlin erst 1984 ins Leben gerufen. Die Mikrozensus-Daten beginnen eigentlich schon in den späten 1950er Jahren, stehen der Wissenschaft aber als Scientific Use Files leider nur lückenhaft ab 1973 zur Verfügung. Hinzu kommt, dass die PanelInformation im Mikrozensus in Deutschland im Gegensatz zu den entsprechenden britischen und französischen Datensätzen nicht generell freigegeben wird. Obwohl es interessant wäre, viele Aspekte des Sozialstaates, die in den 1960er und 1970er Jahren eingeführt worden sind, anhand ihrer Einführung mit alten Daten im Rahmen eines Kontrollgruppenvergleichs zu evaluieren, fehlt es leider fast immer an geeignetem Datenmaterial. Wir haben in diesem Beitrag daher vornehmlich das SOEP verwendet und von daher Reformen der 1990er Jahre evaluiert.

Die Hauptprojekte dieses Forschungsvorhabens, die bereits veröffentlicht sind, evaluieren die Effekte von Lohnfortzahlungen im Krankheitsfall und die Erweiterung des

\footnotetext{
${ }^{1}$ Dieser Beitrag fasst zwei Studien zusammen, die Rahmen des DFG Schwerpunktprogramms ,Flexibilisierungspotenziale bei heterogenen Arbeitsmärkten“" entstanden sind.
}

Erziehungsurlaubes auf 36 Monate. In beiden Fällen schreibt der deutsche Sozialstaat den Arbeitgebern vor, den Arbeitnehmern großzügige Rechte zu gewähren: im Falle der Lohnfortzahlung muss der Arbeitgeber dem Arbeitnehmer bis zu 6 Wochen lang 100 Prozent Lohnfortzahlung im Krankheitsfall gewähren. Im Hinblick auf die Regelungen zum Erziehungsurlaub kann ein Arbeitnehmer (meistens ist es die Mutter) bis zu drei Jahre vom Arbeitsplatz abwesend sein, ohne dass ihr gekündigt werden kann. In beiden Fällen stellt die Regelung in Deutschland den im Vergleich zu anderen Industriestaaten mitunter stärksten Eingriff in das freie Marktgeschehen dar.

Die empirischen Methoden zur Evaluation der Reformen sind sogenannte „Kontrollgruppenansätze“. In beiden Fällen werden Reformen, d.h. Änderungen in den Regelungen, als „natürliche Experimente“ genutzt. Da in unserem Fall nur ein Teil der Gesellschaft von den Reformen betroffen war, wenden wir in beiden Studien Differenz-von-DifferenzenSchätzungen an. Dies bedeutet, dass zwei Gruppen jeweils vor und nach der Reform verglichen werden, wobei nur eine der beiden Gruppen von der Reform betroffen war. Empirische Analysen, denen keine Kontrollgruppe zugrunde liegt, können in der Regel nicht sicherstellen, dass beobachtete Effekte wirklich auf die Reform oder Maßnahme zurückzuführen sind oder ob sie nicht auch ohne die Reform stattgefunden hätten (durch andere unbeobachtbare Einflüsse). Ist allerdings eine geeignete Kontrollgruppe vorhanden, so können die Wirkungen der Reform um Einflüsse, die ohnehin stattgefunden hätten, bereinigt und kausale Effekte der Maßnahme geschätzt werden. Im einfachsten Modell muss dabei angenommen werden, dass sich die betroffene Gruppe von Personen ohne die Reform genauso entwickelt hätte wie die nicht betroffene Gruppe (Annahme gemeinsamer Trends; diese Annahme kann jedoch auch gelockert werden, wenn die Gruppen über mehr als zwei Zeiträume beobachtet werden, darauf soll hier aber nicht eingegangen werden).

Im zweiten Kapitel zeigen wir, dass die 100-prozentige Lohnfortzahlung im Krankheitsfall im Vergleich zu einer nur 80-prozentigen Lohnfortzahlung, die Krankheitstage erhöht. Außerdem lassen Einschnitte im Krankengeld Krankenhausaufenthalte signifikant sinken. Gleichzeitig ändert sich jedoch der subjektiv eingeschätzte Gesundheitszustand nicht. Im dritten Kapitel kommen wir zu dem Ergebnis, dass der lange Erziehungsurlaub auch Kosten für die Betroffenen hat, denen er nutzen soll: junge Frauen erhalten durch den langen Erziehungsurlaub weniger vom Arbeitgeber angeordnete Weiterbildung und kompensieren dies nur zum Teil durch Weiterbildung auf Eigeninitiative.

\section{Lohnfortzahlung im Krankheitsfall und Fehlzeiten}

Verglichen mit den USA sind die Fehlzeiten in Deutschland relativ hoch. Empirische Studien zeigen, dass Fehlzeiten mit 
finanziellen Anreizen oder mit der Sicherheit des Arbeitsplatzes korrelieren (siehe u.a. Riphahn 2004; Riphahn und Thalmaier 2001). Ichino und Riphahn (2005) nutzen die Probezeit als natürliches Experiment und zeigen, dass mit deren Ablauf die Fehlzeiten für Personen in Deutschland und Italien signifikant steigen, da sie in der Regel nun in ein unbefristetes Arbeitsverhältnis übergehen. Für Schweden nutzen Henrekson und Persson (2004) Zeitreihen-Daten von 1955-1999. Sie zeigen, dass mehrere Reformen die Lohnfortzahlung im Krankheitsfall mehr oder weniger großzügig regelten und dass Fehlzeiten jeweils damit korrelieren. So waren in Zeiten großzügiger Regelungen zur Lohnfortzahlung im Krankheitsfall die Fehlzeiten höher und umgekehrt. Johansson und Palme (2002, 2005) zeigen mit Hilfe von Personen-Daten für Schweden anhand eines natürlichen Experiments, dass eine Reform zur Lohnfortzahlung im Krankheitsfall die nur für Arbeiter zutraf (im Gegensatz zu Angestellten) auch nur einen Einfluss auf die Fehlzeiten von Arbeitern hatte. Ein Ländervergleich von Osterkamp (2002) ergibt, dass in Ländern mit großzügigeren Regelungen zur Lohnfortzahlung im Krankheitsfall höhere Fehlzeiten zu beobachten sind, während sie in Ländern mit strengeren Regelungen deutlich niedriger sind.

In Deutschland ist die Lohnfortzahlung im Krankheitsfall im internationalen Vergleich sehr großzügig. Alle Arbeitnehmer erhalten 100 Prozent Lohnfortzahlung für die ersten sechs Wochen einer Krankheit. Danach zahlen die Krankenkassen ein Krankengeld von ca. 70 bis 80 Prozent des Lohns. Diese Regelungen wurden in den 1990er Jahren geändert. Zum 1. Oktober 1996 sollten Arbeitnehmer gesetzlich nur noch 80 Prozent Lohnfortzahlung im Krankheitsfall erhalten. Nach dem Regierungswechsel im Jahr 1998 wurde die gesetzlich geregelte Lohnfortzahlung im Krankheitsfall zum 1. Januar 1999 wieder auf 100 Prozent festgelegt, so dass alle Arbeitnehmer wieder 100 Prozent Lohnfortzahlung in den ersten 6 Wochen der Abwesenheit vom Arbeitsplatz durch Krankheit erhielten.

Die Änderungen in der Lohnfortzahlung können als natürliches Experiment auf Basis der SOEP-Daten evaluiert werden und Aufschluss darüber geben, wie Arbeitnehmer auf Kürzungen der Lohnfortzahlung reagieren (siehe Puhani und Sonderhof 2010). Hierzu müssen die Beschäftigten in eine Gruppe von Betroffenen (treatment group) und eine Kontrollgruppe (control group) eingeteilt werden. Diese Einteilung der Arbeitnehmer ist möglich, da die Reform zur Kürzung der gesetzlich geregelten Lohnfortzahlung im Krankheitsfall auf 80 Prozent aufgrund von Tarifverträgen de facto fast nur Arbeitnehmer ohne Tarifvertrag betraf. Die meisten Arbeitnehmer mit Tarifvertrag erhielten somit weiterhin 100\% Lohnfortzahlung aufgrund tariflicher Regelungen. ${ }^{2}$ Aus diesem Grund kann eine Evaluation der Re-

\footnotetext{
${ }^{2}$ Die Gruppe der Betroffenen und die Kotrollgruppe können nicht ganz scharf getrennt werden. Wenige Arbeitnehmer erhielten laut ihrem Ta-
}

form mit Hilfe von Differenz-von-Differenzen Schätzungen durchgeführt werden. Die Kontrollgruppe der Personen mit Tarifvertrag bekam die ganze Zeit volle Lohnfortzahlung im Krankheitsfall, während die Gruppe der Betroffenen (Arbeitnehmer ohne Tarifvertrag) in den Jahren 1997 und 1998 nur 80 Prozent erhielten.

Deskriptive Ergebnisse zeigen bereits, dass es deutliche Unterschiede zwischen der Gruppe der Betroffenen und der Kontrollgruppe gibt (Abb. 1). Während die Krankheitstage der Kontrollgruppe im Reformzeitraum (1997/1998) sogar leicht anstiegen, sanken sie für die Arbeitnehmer ohne Tarifvertrag, die in diesem Zeitraum nur 80 Prozent Lohnfortzahlung im Krankheitsfall erhielt, von 8,1 Tagen vor der Reform auf 6,4 Tage (entspricht etwa 21 Prozent) während der Reform und wieder auf 9,3 Tage (entspricht einem Anstieg um ca. 45 Prozent) nach der Rücknahme der Reform. Auffällig ist darüber hinaus, dass die Kontrollgruppe der Tarifbeschäftigten ohnehin deutlich höhere Fehlzeiten hat als die von der Reform betroffene Gruppe.

In einer Differenz-von-Differenzen Schätzung werden die Unterschiede in den Krankheitstagen vor und während der Reform gebildet (Tab. 1, letzte Spalte) und zwischen Personen ohne und mit Tarifvertrag (vierte Zeile). Es zeigt sich, dass Personen mit Tarifvertrag während der Reform 0,6 Tage mehr gefehlt haben als vor der Reform, während Personen ohne Tarifvertrag 1,7 Tage seltener gefehlt haben. Der Unterschied zwischen beiden Gruppen ist 2,3 Tage (fett gedruckt) und damit der Differenz-von-Differenzen Schätzwert. ${ }^{3}$ Die gleiche Zahl ergibt sich als Differenz zwischen dem Unterschied zwischen Personen ohne und mit Tarifvertrag während der Reform ( $-5,6$ Tage) und vor der Reform (-3,3 Tage). Der obere Teil von Tab. 1 bedeutet folglich, dass im Vergleich zu der Zeit vor der Reform, Personen ohne Tarifvertrag während der Reform 2,3 Tage seltener gefehlt haben als Personen mit Tarifvertrag. Der aufgrund der Reform geschätzte Rückgang der Fehlzeiten ist somit größer als der beobachtete Rückgang von 1,7 Tagen bei der betroffenen Gruppe, da die Kontrollgruppe sogar 0,6 Tage häufiger

rifvertrag nur 80 Prozent Lohnfortzahlung im Krankheitsfall. Allerdings berichtet Deutscher Bundestag (1998), dass etwa 80 Prozent Beschäftigten aufgrund von bestehenden Tarifverträgen volle Lohnfortzahlung im Krankheitsfall erhielten, was sich etwa mit dem Anteil der Beschäftigten mit Tarifvertrag deckt. Sollten einige Arbeitnehmer mit Tarifvertrag dennoch nur 80 Prozent Lohnfortzahlung erhalten haben, verzerrt dies unsere Ergebnisse Richtung Null (attenuation bias), so dass der wahre Effekt in diesem Fall sogar noch größer sein kann als nach unserer Schätzung.

${ }^{3}$ Falls kranke Arbeitnehmer ihre Kollegen anstecken, ist dies in der Schätzung bereits berücksichtigt, falls diese Personen vom Arbeitsplatz fernbleiben (wir nehmen an, dass Kollegen im unmittelbaren Umfeld der gleichen Gruppe angehören, d.h. dass entweder alle Teil der Betroffenen- oder der Kontrollgruppe sind). Da wir uns auf Erwerbstätige beschränken, sind Effekte auf Kündigungen nicht berücksichtigt. Aufgrund des hohen Kündigungsschutzes in Deutschland sollten diese Effekte jedoch gering sein. 
Abb. 1 Fehlzeiten der Arbeitnehmer ohne und mit Tarifvertrag (betroffene Gruppe und Kontrollgruppe)

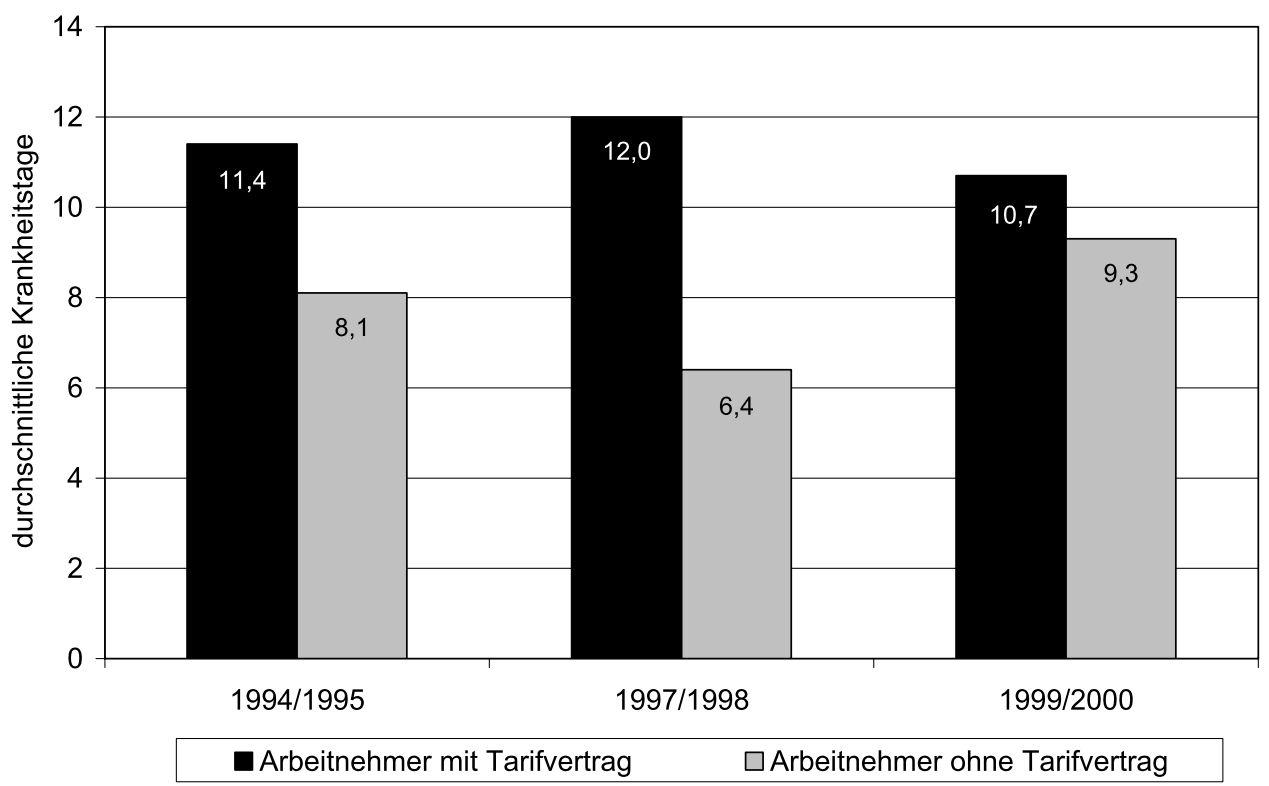

Quelle: P.A. Puhani; K. Sonderhof (2010).

Tab. 1 Differenz-von-Differenzen ohne Kontrollvariablen

\begin{tabular}{lccc}
\hline & $1994 / 1995$ (vor Reform) & $1997 / 1998$ (während Reform) & Differenz (während/vor) \\
\hline Arbeitnehmer mit Tarifvertrag & 11,4 & 12,0 & 0,6 \\
Arbeitnehmer ohne Tarifvertrag & 8,1 & 6,4 & $-1,7$ \\
Differenz (ohne/mit Tarifvertrag) & $-3,3$ & $-5,6$ & $\mathbf{- 2 , 3}$ \\
\hline & $1997 / 1998$ (während Reform) & $1999 / 2000$ (nach Reform) & Differenz (während/nach) \\
\hline Arbeitnehmer mit Tarifvertrag & 12,0 & 10,7 & 1,3 \\
Arbeitnehmer ohne Tarifvertrag & 6,4 & 9,3 & $-2,9$ \\
Differenz (ohne/mit Tarifvertrag) & $-5,6$ & $-1,4$ & $\mathbf{- 4 , 2}$ \\
\hline
\end{tabular}

Quelle: P.A. Puhani; K. Sonderhof (2010).

krank war als vor der Reform. Diese 0,6 Tage höheren Fehlzeiten werden als „Maßstab“ verwendet, wie sich die Fehlzeiten ohne die Reform entwickelt hätten, die aufgrund von externen Einflüssen wie zum Beispiel Wetter von Jahr zu Jahr variieren können. Im Vergleich zu diesem Maßstab haben Angestellte ohne Tarifvertrag 2,3 Tage seltener gefehlt. Die kritische Annahme bei dieser Betrachtung ist, dass sich beide Gruppen im Zeitverlauf gleichermaßen entwickelt hätten, wenn die Reform nicht stattgefunden hätte, also dass es bei der betroffenen Gruppe im Zeitraum 1997/1998 ohne die Reform auch einen leichten Anstieg der Krankheitstage gegeben hätte und anschließend einen Rückgang. Das heißt, das Niveau der Fehlzeiten in den beiden Gruppen darf generell unterschiedlich sein, aber dieser Unterschied müsste im hypothetischen Fall, dass keine Reform stattgefunden hätte, über die Zeit konstant geblieben sein. Der untere Teil von Tab. 1 zeigt die Ergebnisse der Rücknahme der Reform und kann folgendermaßen interpretiert werden: Im Vergleich zu der Zeit als die Reform zur Lohnkürzung in Kraft war, haben die Betroffenen (ohne Tarifvertrag) nach der Rücknahme der Reform 4,2 Tage häufiger gefehlt als die Kontrollgruppe (Personen mit Tarifvertrag).

In Tab. 1 wird nicht für beobachtbare Charakteristika wie Alter, Ausbildung, Geschlecht usw. kontrolliert. Wird der Differenz-von-Differenzen Ansatz allerdings in einer Regressionsgleichung mit Kontrollvariablen geschätzt (Arbeitslosenquote im Bundesland, Stundenlohn, Familienstand, Geschlecht, Schulbildung, Ausbildung, Staatsangehörigkeit, Berufs- und Firmencharakteristika, Dummy für Ost- bzw. Westdeutschland, Gesundheitszustand und Zufriedenheit mit dem Gesundheitszustand), so kann der deskriptive Eindruck bestätigt werden. Im Vergleich zu der Kontrollgruppe waren die Krankheitstage der Angestellten ohne Tarifvertrag im Alter zwischen 20 und 55 Jahren im Zeit- 
raum der Reform je nach Schätzmethode 2,3 bis 2,9 Tage geringer (im Vergleich zu der Zeit vor und nach der Rücknahme der Reform). Eine separate Schätzung des Effekts der Einführung und der Rücknahme der Reform zur Lohnkürzung im Krankheitsfall zeigt, dass durch die Einführung die Krankheitstage der betroffenen Personen weniger gesunken sind als sie nach der Rücknahme der Reform wieder angestiegen sind, jeweils im Vergleich zu der Kontrollgruppe der Personen mit Tarifvertrag, für die sich hinsichtlich der Lohnfortzahlung im Krankheitsfall nichts geändert hat. So hat die Einführung der Lohnkürzung im Krankheitsfall zu einem Rückgang der Fehlzeiten von 2 bis 2,3 Tagen bei den betroffenen Personen geführt, während nach der Rücknahme der Reform die Fehlzeiten um 2,4 bis 4,5 Tage angestiegen sind (jeweils abhängig von der Schätzmethode). Auch diese Ergebnisse stehen im Einklang mit den deskriptiven Beobachtungen in Tab. 1.

Es stellt sich nun die Frage, ob der Rückgang der Krankheitstage während der nur 80-prozentigen Lohnfortzahlung im Krankheitsfall darauf zurückzuführen ist, dass einige Personen krank zur Arbeit kamen, oder ob sie vor der Reform und nach Rücknahme der Reform einfach nicht zur Arbeit kamen, obwohl sie nicht krank waren. Wie sich zeigt, scheint die Situation etwas komplizierter zu sein. Um dieser Frage nachzugehen, wird zunächst der selbst eingeschätzte Gesundheitszustand als weitere Ergebnisvariable zur Evaluation der Reform betrachtet. Unsere Analysen zeigen, dass die Reduzierung der Lohnfortzahlung keinen nachweislichen Einfluss auf den selbst eingeschätzten Gesundheitszustand hatte, was bedeutet, dass sich die Arbeitnehmer während der Reform trotz geringerer Fehlzeiten genauso gesund oder krank fühlten wie vor oder nach Rücknahme der Reform. ${ }^{4}$

In weiteren Analysen stellen wir uns die Frage, ob die Kürzung der Lohnfortzahlung im Krankheitsfall auch einen Einfluss auf die Nutzung der Gesundheitsversorgung hatte. Um das zu untersuchen, werden die Effekte der Reform auf die Anzahl der Arztbesuche und auf die Krankenhausaufenthalte sowie die Dauer der Aufenthalte als abhängige Variablen geschätzt. Empirisch zeigt sich, dass die geringeren Fehlzeiten der von der Lohnkürzung betroffenen Personen mit signifikant kürzeren Krankenhausaufenthalten einhergehen. Das bedeutet, betroffene Personen haben während der Reform kürzere Krankenhausaufenthalte (verbunden mit geringeren Fehlzeiten) und schätzen ihren Gesundheitszustand dennoch genauso ein wie vor der Reform. Eine Interpretation dieser Ergebnisse könnte sein, dass durch die Reduktion der Lohnfortzahlung im Krankheitsfall die ineffiziente

\footnotetext{
${ }^{4}$ Falls Arbeitnehmer während der Reform krank zur Arbeit gekommen wären und sich somit der Genesungsprozess verzögert hätte, sollte sich dies ebenfalls in einer Verschlechterung des durchschnittlichen Gesundheitszustandes in der von der Reform betroffenen Gruppe ausdrücken.
}

Nutzung des Gesundheitssystems verringert wurde. Dies ist nicht unplausibel, da die Anzahl der Krankenhaustage pro Person in Deutschland in den betrachteten Jahren im Vergleich zu den USA oder Großbritannien relativ hoch war, ohne dass Deutschland eine deutlich höhere Lebenserwartung als diese Länder aufweisen konnte.

Laut OECD betrugen die Gesundheitsausgaben im Jahre 1995, also kurz vor der Kürzung der Lohnfortzahlung im Krankheitsfall, in Deutschland 10,1\% des Bruttoinlandsprodukts, mehr als in Großbritannien (6,9\%) aber weniger als in den USA (13,3\%). Die Lebenserwartung bei Geburt war in diesen drei Ländern jedoch ungefähr gleich (zwischen 75,7 und 76,7 Jahren). Die Anzahl der Arztbesuche pro Jahr war in Deutschland am höchsten $(6,4)$, gefolgt von Großbritannien $(6,1)$ und den USA $(3,3)$; zum Jahr 2003 wurde dieser Unterschied noch größer, mit Werten von jeweils 7,6, 5,2 und 3,9. Die durchschnittliche Anzahl der Krankenhausaufenthalte pro Person war 0,18, 0,21 und 0,12 und die durchschnittliche Dauer stationärer Aufenthalte betrug 11,4, 7,1 und 6,5 Tage in Deutschland, Großbritannien und den USA. Damit wies Deutschland bei Weitem die längsten durchschnittlichen Krankenhausaufenthalte auf. Die Reduzierung der Lohnfortzahlung im Krankheitsfall hat laut unseren Ergebnissen dazu beigetragen, diese Lücke etwas zu verringern, ohne den subjektiv empfundenen Gesundheitszustand der betroffenen Personen zu beeinträchtigen.

Zusammenfassend ist die höhere Anzahl der Krankheitstage bei 100-prozentiger Lohnfortzahlung im Krankheitsfall auch dadurch erklärbar, dass deutsche Arbeitnehmer bei Krankheit längere Zeit das Gesundheitssystem (inkl. Krankenhausaufenthalte) nutzen als bei 80-prozentiger Lohnfortzahlung, obwohl dies keinen deutlich nachweisbaren Nutzen auf die subjektive Gesundheit hat. Die längeren Krankenhausaufenthalte sind auch dadurch erklärbar, dass zum damaligen Zeitpunkt für Krankenhäuser finanzielle Anreize bestanden, Patienten eher länger als kürzer in stationärer Behandlung zu behalten. Zu dem Zeitpunkt der Reform wurden Krankenhäuser mehr oder weniger nach Länge der Krankenhausaufenthalte der Patienten bezahlt, so dass es aus ihrer Sicht ökonomisch sinnvoll erschien, die Patienten möglichst lange zu behandeln. Ab 2004 wurden die Abrechnungen der Krankenhäuser auf Fallpauschalen umgestellt, das heißt je nach Krankheit eine bestimmte fixe Summe, so dass Anreize geschaffen wurden, Patienten möglichst kurz im Krankenhaus zu behalten. Für die Patienten ergeben sich grundsätzlich durch die Umstellung auf Fallpauschalen keine Anreize für einen längeren oder kürzeren Krankenhausaufenthalt. Seit 2004 müssen Patienten im Krankenhaus allerdings pro Behandlungstag 10 Euro Selbstbeteiligung zahlen. Diese Änderung könnte zur Verringerung der Länge der Krankenhausaufenthalte beitragen, fand allerdings nicht im Untersuchungszeitraum dieses Beitrags statt. 


\section{Weiterbildungschancen von jungen Frauen nach der Verlängerung des Erziehungsurlaubes}

Im Rahmen der Evaluation sozialpolitischer Reformen in Deutschland wird in diesem Abschnitt die Verlängerung des Erziehungsurlaubes von 18 auf 36 Monate auf die Weiterbildungschancen junger Frauen als natürliches Experiment untersucht. An dieser Stelle werden die Ergebnisse der Untersuchung von Puhani und Sonderhof (2011) zusammengefasst. Deutschland gehört weltweit zu den Ländern, die Müttern und Vätern den längsten Erziehungsurlaub gewähren. Seit 1992 können Mütter bis zu drei Jahre lang ihren Arbeitsplatz verlassen und haben anschließend ein Anrecht auf eine vergleichbare Position bei ihrem früheren Arbeitgeber. Bei den Mutterschutzregelungen gibt es zwischen den industrialisierten Ländern zum Teil erhebliche Unterschiede. Zum Beispiel haben Mütter in den USA erst seit 1993 das Recht innerhalb von 12 Wochen nach der Geburt an ihren Arbeitsplatz zurückzukehren, zuvor gab es keine bundesstaatlich einheitlichen Regelungen. Doch haben die großzügigen Regelungen in Deutschland nicht nur Vorteile für Mütter und jungen Frauen. Es wäre anzunehmen, dass Arbeitgeber weniger in das Humankapital junger Frauen investieren (und ihnen somit weniger Weiterbildungsmöglichkeiten bieten), wenn sie wissen, dass diese den Arbeitsplatz im Fall eines Kindes für bis zu drei Jahre lang verlassen können.

Die oben genannte Reform in Deutschland wird als natürliches Experiment evaluiert, indem die Wahrscheinlichkeit von jungen Frauen an einer Weiterbildung teilzunehmen vor und nach der Reform mit Weiterbildungswahrscheinlichkeiten von geeigneten Kontrollgruppe verglichen wird. Dabei sind unterschiedliche Kontrollgruppen denkbar: Zum einen eignen sich ältere Frauen, da aus Sicht des Arbeitgebers hier die Möglichkeit einer Schwangerschaft unwahrscheinlicher ist. ${ }^{5}$ Als zweite Kontrollgruppe werden junge Männer herangezogen. Diese sind jungen Frauen im Hinblick auf den bisherigen Karriereverlauf ähnlicher und haben zum Zeitpunkt der Reform - 1992 - sehr selten Erziehungsurlaub genommen. ${ }^{6}$ Als letztes Kontrollgruppendesign werden junge und ältere Männer sowie ältere Frauen in einer Differenzvon-Differenz-von-Differenzen-Schätzung verwendet. Die Ergebnisse basieren hauptsächlich auf dem Datensatz „Berichtssystem Weiterbildung (BSW)“. Hier stehen Daten von 1988 und 1994 als Beobachtungen vor und nach der Reform zur Verfügung. Da die Daten vor der Reform bereits vier Jahre vor der Verlängerung des Erziehungsurlaubes auf

\footnotetext{
${ }^{5}$ Der Anteil der Frauen, die bei der Geburt über 40 Jahre alt sind liegt bei etwa 2 Prozent.

${ }^{6}$ Seit 1986 können auch Väter in Erziehungsurlaub gehen, allerdings machten zum Zeitpunkt der Reform zur Verlängerung des Erziehungsurlaubes weniger als 1,5 Prozent der Männer davon Gebrauch.
}

36 Monate erhoben wurden, ist nicht zu erwarten, dass die Reform von Seiten des Arbeitgebers oder der beschäftigten Frauen antizipiert wurde.

Unsere Differenz-von-Differenzen Analysen, die auch gruppenspezifische Trends kontrollieren, zeigen, dass die Wahrscheinlichkeit, an einer vom Arbeitgeber angeordneten beruflichen Weiterbildung teilzunehmen, sich für junge Frauen in den Jahren nach der Verlängerung des Erziehungsurlaubes deutlich um ein Drittel oder gar die Hälfte verringert hat. Dies lässt sich dahingehend interpretieren, dass Arbeitgeber auf die Gesetzesänderung reagieren und weniger in das Humankapital junger Frauen investieren, wenn diese anschließend die Möglichkeit haben, ihren Arbeitsplatz für drei Jahre zu verlassen.

Kürzungen bei der Weiterbildung von jungen Frauen werden besonders deutlich, wenn die Art der Weiterbildung berücksichtigt wird. Dazu wird die gesamte berufliche Weiterbildung in „Weiterbildung auf Anordnung des Arbeitgebers" und „Weiterbildung aus Eigeninitiative“ unterteilt. Die Weiterbildung auf Anordnung des Arbeitgebers ist für junge Frauen nach der Verlängerung des Erziehungsurlaubes zwischen 30 und 50 Prozent gesunken. Im Gegenzug nahmen zwar die Eigenaktivitäten der Frauen auf dem Gebiet der Weiterbildung um bis zu 40 Prozent zu, trotzdem steht per Saldo ein Rückgang der Weiterbildungswahrscheinlichkeit um bis zu 20 Prozent. Junge Frauen scheinen demnach zu realisieren, dass sie weniger Weiterbildungsmöglichkeiten von Seiten des Arbeitgebers bekommen und kompensieren es, indem sie sich selbst verstärkt um Weiterbildungen kümmern.

Auffallend ist darüber hinaus, dass Arbeitgeber nach der Verlängerung des Erziehungsurlaubes auch die Weiterbildung von jungen Frauen ohne Kinder reduzieren, sofern diese im gebärfähigen Alter sind und somit von der dreijährigen „Babypause“ mit Beschäftigungsschutz Gebrauch machen könnten. Dies liegt darin begründet, dass ein Arbeitgeber im Vorfeld nicht wissen kann, welche Frauen aufgrund von möglichen Erziehungsurlauben ausfallen werden. Von daher sind auch Frauen ohne Kinder negativ von der Gesetzesänderung betroffen.

Es ist möglich, dass der in Deutschland vergleichsweise sehr lange Erziehungsurlaub positive Effekte in anderen Bereichen für Mütter und Kinder hat. Dustmann und Schönberg (2008) zeigen jedoch, dass ein längerer Erziehungsurlaub weder zu höheren Bildungsabschlüssen noch zu höheren Löhnen der betroffenen Kinder in Deutschland geführt hat. Zumindest in dieser Hinsicht konnte kein positiver Effekt auf das Wohl des Kindes festgestellt werden. Für die Mütter scheint ein langer Erziehungsurlaub allerdings auch eher einen negativen Effekt auf Erwerbstätigkeit und Löhne zu haben. Ondrich et al. (2003) und Lalive und Zweimüller (2005) zeigen für Deutschland bzw. Österreich, dass ein längerer Erziehungsurlaub das kurzfristige Arbeitsangebot sin- 
ken lässt. Schönberg und Ludsteck (2007) finden auch längerfristige Auswirkungen auf das Arbeitsangebot von Frauen, während Ruhm (1998) in einer vergleichenden Studie für europäischer Länder zeigt, dass längere Erziehungsurlaubszeiten zwar mit niedrigeren Löhnen des Erziehungsurlaubers (meistens Mütter) korrelieren, allerdings teilweise auch mit einer erhöhten Frauenerwerbstätigkeit.

Ein Argument für lange Erziehungsurlaubszeiten wäre, dass durch die Möglichkeit eine längere Auszeit zu nehmen die Geburtenrate steigen könnte. In Deutschland scheint dies allerdings nicht den gewünschten Effekt gehabt zu haben. Seit der schrittweisen Verlängerung des Erziehungsurlaubes im Jahr 1986 ging die Geburtenrate pro Frau tendenziell zurück anstatt anzusteigen, wobei natürlich nicht klar ist, wie sich die Geburtenraten ohne die Reformen entwickelt hätte. Abschließend kann zusammengefasst werden, dass es durch die Verlängerung des Erziehungsurlaubes zu negativen Effekten für Mütter im Berufsleben gekommen ist (geringere Löhne, weniger Weiterbildung), während kein positiver Effekt im Hinblick auf das Wohl des Kindes gefunden werden konnte.

\section{Schlussfolgerungen}

Unser Beitrag zeigt, dass beide hier evaluierten Eingriffe des Staates in den Arbeitsmarkt neben den gewollten positiven unterstützenden Effekten für Kranke (im einen Fall) und junge Frauen (im anderen Fall) auch negative Effekte haben. Die Verpflichtung des Arbeitgebers, einem abwesenden Arbeitnehmer 100 Prozent statt 80 Prozent des Lohnes zu bezahlen, erhöht die durchschnittlichen Abwesenheitstage vom Arbeitplatz von ca. 6 auf ca. 8 Tage (Abweichungen je nach Spezifikation; einige Schätzungen liefern sogar noch etwas größere Effekte), ohne das subjektive Gesundheitsempfinden der Arbeitnehmer nachweislich negativ zu beeinträchtigen. Die Verpflichtung des Arbeitgebers, Eltern bis zu 3 Jahren den Arbeitsplatz auch bei Abwesenheit zu garantieren, reduziert die von Seiten des Arbeitgebers angeordnete Weiterbildung für junge Frauen.

\section{Executive Summary}

The welfare state (or "social market economy" as it is called in German) is experiencing significant challenges. Continuing reform efforts in the fields of health, pensions, unemployment, family and other benefits reflect the broad range of problems facing policy makers. Low birth rates combined with low employment rates are part of the key determinants that make Germany's welfare state so fragile. Opponents to major reform of the welfare state in its current form often ignore the many changes that occurred in the system during the past decades.
Since the late 1950s, the economic success of the fledgling German democratic state was increasingly used for an expansion of social benefits. For example, unemployment benefits were increased, parental leave was extended and sick pay was regulated to equal the wage from the first day of sickness. Besides the politically desired distributional effects, the expansion of the welfare state also provided incentives related to labor supply and labor demand. There is, however, little research on these effects, probably due to the lack of good data at the personal level for earlier periods.

The aim of this paper is to show two examples of estimating causal effects of social policy reforms on the labor market, using econometric analyses based on personal data.

The main analyses of this contribution evaluate the effect of extension of parental leave on job-related training and the effects of sick pay reform on absence and on health related outcomes. In both cases, German law gives very generous rights to employees when compared to other industrialized countries. A parent (mostly the mother) can take 36 months of parental leave without losing employment protection. Sick pay has to be paid as 100 percent of the wage by the employer for up to 6 weeks of absence from work. The reform we investigate reduced sick pay from 100 to 80 percent. We are also able to evaluate the repeal of the reform about 2 years later when the federal government changed, so that for this reform, we can estimate switch on and switch off effects.

Both our analyses are methodologically based on difference-in-differences approaches. The control group designs used to evaluate the extension of maternity benefits compares young women (the treatment group) with control group designs involving young men, older women and young and older men together with older women (in this case a difference-in-difference-in differences analysis). For the evaluation of sick pay reform, the treatment group are workers not covered by collective bargaining (they were affected by the reform), the control group are workers covered by collective bargaining (as collective bargaining contracts mostly compensated the change in federal law by specifying that workers still be paid 100 percent of their wage, so what was previously the federal law, was now enforced by the collective bargaining contract).

For the extension of maternity leave from between 10/18 to 36 months, we find that it reduced job-related training for young women. This is especially true for employer-provided training, which fell by between 30 and 50 percent. As a reaction to that, young women increased job-related training on their own initiative by up to 40 percent. Hence, a representative estimate is that young women's job-related training was reduced by about 20 percent. Remarkably, even women without children seem to have been affected by the extension of parental leave, because their employer-provided training decreased as well. This can be explained by the fact that 
employers might expect women without children to become mothers and hence reduce their human capital investments in them.

As to the sick pay reform, we find that during the period when sick pay was 80 instead of 100 percent the number of days of absence from work was about 2-days lower for the average member of the treatment group. At the same time, we cannot detect any negative effects on subjective health indicators, although the reform decreased the average number of days spent in hospital by almost half a day. Hence we conclude that the very generous sick pay regime in Germany might cause inefficient use of the health care system.

\section{Zusammenfassung:}

Die soziale Marktwirtschaft der Bundesrepublik Deutschland steht vor großen Herausforderungen. Anhaltende Reformbemühungen in den Bereichen Gesundheit, Alterssicherung, Lohnersatzleistungen und Familienpolitik verdeutlichen die Breite der Problematik. Geringe Geburtenzahlen und niedrige Beschäftigungsquoten gehören zu den wesentlichen Determinanten der Fragilität des Sozialstaates in seiner heutigen Form. Bekenntnisse der politischen Entscheidungsträger zum System „soziale Marktwirtschaft" lassen häufig die Dynamik außer Acht, die der Sozialstaat in den letzten Jahrzehnten entwickelt hat.

Seit den späten 1950er Jahren wurde der wirtschaftliche Erfolg des jungen deutschen Staates zunehmend auch für eine Intensivierung des sozialen Ausgleiches genutzt. Beispiele sind höhere Leistungen für Arbeitslose, ein verlängerter Erziehungsurlaub oder die schrittweise Abschaffung von Karenztagen. Neben der politisch gewünschten Verteilungswirkung entstanden durch die genannten Reformen auch Anreize bezüglich des Arbeitsangebots und der Arbeitsnachfrage, die jedoch in früheren Jahrzehnten (vermutlich aufgrund der guten wirtschaftlichen Lage und auch der mangelnden Verwertbarkeit von Personendaten) nicht erforscht wurden.

Ziel dieses Beitrags ist es zu illustrieren, wie auf Basis von Personendaten mittels ökonometrischer Analysen kausale Effekte sozialstaatlicher Reformen auf den Arbeitsmarkt evaluiert werden können. In dem Zusammenhang untersucht dieser Beitrag den Effekt der Verlängerung des Erziehungsurlaubes auf die Wahrscheinlichkeit, an einer Weiterbildung teilzunehmen. Zudem wird untersucht, ob eine Reform der Lohnfortzahlung im Krankheitsfall einen Einfluss auf die Fehlzeiten und die subjektiv eingeschätzte Gesundheit der Betroffenen hat. In beiden Fällen sieht das deutsche Recht für Arbeitnehmer großzügigere Regelungen vor, als das in vergleichbaren Industrienationen der Fall ist: Ein Elternteil (meist ist es die Mutter) kann bis zu 36 Monate in Elternzeit gehen und hat anschließend ein Anrecht auf eine vergleichbare Stelle bei dem früheren Arbeitgeber. Im Falle der Lohnfortzahlung im Krankheitsfall muss der Arbeitgeber dem Arbeitnehmer bis zu 6 Wochen lang 100 Prozent Lohnfortzahlung im Krankheitsfall gewähren. Die untersuchte Reform reduzierte die Lohnfortzahlung im Krankheitsfall auf 80 Prozent, wobei die Kürzung bereits zwei Jahre später wieder zurückgenommen wurde, so dass Effekte der Einführung und der Rücknahme der Reform separat geschätzt werden können.

Bei beiden Untersuchungen werden methodisch sogenannte Kontrollgruppenansätze, also Differenz-von-Differenzen Schätzungen verwendet. Bei der Verlängerung des Erziehungsurlaubes wird die Weiterbildungswahrscheinlichkeit junger Frauen (die Gruppe der Betroffenen) mit der verschiedener Kontrollgruppen verglichen. Zum einen werden junge Männer und ältere Frauen in Differenz-vonDifferenzen Schätzgungen als Kontrollgruppe verwendet, zum anderen die Gruppe der Männer und älteren Frauen zusammen in einer Differenz-von-Differenz-von-Differenzen Schätzung. Bei der Untersuchung der Kürzung der Lohnfortzahlung im Krankheitsfall stellen Angestellte ohne Tarifvertrag die betroffene Gruppe dar (da nur für sie die Kürzung galt), während alle Angestellten mit Tarifvertrag in der Kontrollgruppe sind. Sie waren nicht von der Reform betroffen, da in den meisten Tarifverträgen bereits im Vorfeld eine 100-prozentige Lohnfortzahlung ausgehandelt wurde.

Im Hinblick auf die Reform zur Verlängerung des Erziehungsurlaubes von 10 bzw. 18 Monate auf 36 Monate zeigen wir, dass junge Frauen nach der Verlängerung signifikant weniger berufliche Weiterbildung erhalten. Dies trifft besonders auf Weiterbildungen zu, die vom Arbeitgeber angeordnet wurden, welche nach der Reform um 30 bis 50 Prozent seltener stattfanden. In Folge darauf nahm die Weiterbildung aus Eigeninitiative bei jungen Frauen um bis zu 40 Prozent zu. Insgesamt ist die Weiterbildungswahrscheinlichkeit junger Frauen allerdings um rund 20 Prozent zurückgegangen. Bemerkenswert ist darüber hinaus, dass sogar die Wahrscheinlichkeit, an einer beruflichen Weiterbildung teilzunehmen, bei jungen Frauen ohne Kinder abgenommen hat. Dies kann dadurch erklärt werden, dass aus Sicht des Arbeitgebers bei jungen Frauen generell das Risiko besteht, dass sie in Zukunft temporär ausfallen, was dazu führen kann, dass Arbeitgeber im Vorfeld weniger in ihr Humankapital investieren.

Bei der Reform zur Kürzung der Lohnfortzahlung im Krankheitsfall kommen wir zu dem Ergebnis, dass während der Zeit der geringeren Lohnfortzahlung die Anzahl der Krankheitstage bei der betroffenen Gruppe um etwa 2 Tage im Jahr gesunken ist. Gleichzeitig finden wir keine negativen Effekte auf das subjektive Gesundheitsempfinden, obwohl im Reformzeitraum auch die Anzahl der Tage im Krankenhaus im Durchschnitt um fast einen halben Tag zurückgegangen ist. Aus diesem Grund folgern wir, dass die großzügigen Regelungen der Lohnfortzahlung im Krankheitsfall 
in Deutschland zu einer ineffizienten Nutzung des Gesundheitssystems führen können.

Acknowledgements Part of this research was supported by German Research Foundation (DFG) within the project 'Labour Market Effects of Social Policy' which is part of the research initiative 'Flexibility in Heterogeneous Labour Markets'. We are grateful to the editor, Bernd Fitzenberger, several anonymous referees, Christian Dustmann, John Heywood, Jeff Smith, Uta Schönberg, Alfonso Sousa-Poza, Marie Waller, Fan $\mathrm{Wu}$ and many conference participants for helpful comments. All remaining errors are our own.

\section{Literatur}

Deutscher Bundestag: Drucksache 14/45, S. 17, verfügbar im Internet (27.12.2010) unter http://dipbt.bundestag.de/dip2/btd/14/000/ 1400045.pdf (1998)

Dustmann, C., Schönberg, U.: The effect of expansions in maternity leave coverage on children's long-term outcome. IZA Diskussionspapier No. 3605, Bonn (2008)

Frerich, J.: Sozialpolitik: Das Sozialleistungssystem der Bundesrepublik Deutschland, 3. Aufl. Oldenbourg, München (1996)

Frerich, J., Frey, M.: Handbuch der Geschichte der Sozialpolitik in Deutschland, Bd. 3. Oldenbourg, München (1993)

Henrekson, M., Persson, M.: The effects on sick leave of changes in the sickness insurance system. J. Labor Econ. 22, 87-113 (2004)

Ichino, A., Riphahn, R.T.: The effect of employment protection on worker effort: absenteeism during and after probation. J. Eur. Econ. Assoc. 3, 120-143 (2005)

Johansson, P., Palme, M.: Assessing the effect of public policy on worker absenteeism. J. Hum. Resour. 37, 381-409 (2002)

Johansson, P., Palme, M.: Moral hazard and sickness insurance. J. Public Econ. 89, 1879-1890 (2005)

Lalive, R., Zweimüller, J.: (2005) Does parental leave affect fertility and return-to-work? Evidence from a "true natural experiment". IZA Diskussionspapier Nr. 1613, Bonn

Lampert, H., Bossert, A.: Sozialstaat Deutschland. Vahlen, München (1992)

Lampert, H., Althammer, J.: Lehrbuch der Sozialpolitik, 6. Aufl. Springer, Berlin (2001)
Metzler, G.: Der deutsche Sozialstaat. Vom bismarckschen Erfolgsmodell zum Pflegefall. Stuttgart (2003)

Ondrich, J., Spiess, C.K., Yang, Q., Wagner, G.G.: The liberalization of maternity leave policy and the return to work after childbirth in Germany. Rev. Econ. Househ. 1, 77-110 (2003)

Osterkamp, R.: Dice reports: work lost due to illness-an international comparison. CESifo Forum 4, 36-40 (2002)

Puhani, P.A., Sonderhof, K.: The effects of a sick pay reform on absence and on health-related outcomes. J. Health Econ. 29, 285302 (2010)

Puhani, P.A., Sonderhof, K.: The effects of a parental leave extension on training for young women. J. Popul. Econ. 24, 731-760 (2011)

Riphahn, R.T.: Employment protection and effort among German employees. Econ. Lett. 85, 353-357 (2004)

Riphahn, R.T., Thalmaier, A.: Behavioural effects of probation periods: an analysis of worker absenteeism. Jahrb. Natl.ökon. Stat. 221, 178-201 (2001)

Ruhm, Ch.J.: The economic consequences of parental leave mandates: lessons from Europe. Q. J. Econ. 113, 285-317 (1998)

Schönberg, U., Ludsteck, J.: Maternity leave legislation, female labor supply, and the family wage gap. IZA Diskussionspapier Nr. 2699, Bonn (2007)

Patrick A. Puhani ist Professor für Volkswirtschaftslehre und Direktor des Instituts für Arbeitsökonomik der Wirtschaftswissenschaftlichen Fakultät der Leibniz Universität Hannover. Er ist ebenfalls Research Fellow am Centre for Research and Analysis of Migration (CReAM), University College London (UCL); am Equipe de Recherche sur les Marchés, l'Emploi et la Simulation (ERMES), Université Paris II (Panthéon-Assas); am Forschungsinstitut zur Zukunft der Arbeit (IZA), Bonn; und am Schweizerischen Institut für Empirische Wirtschaftsforschung (SEW), Universität St. Gallen.

Katja Sonderhof studierte Mathematik an der Technischen Universität Darmstadt und der Nanyang Technological University in Singapur. Nach dem Studium war sie wissenschaftliche Mitarbeiterin und Doktorandin der Volkswirtschaftslehre an der TU Darmstadt und der Leibniz Universität Hannover. Im Jahr 2010 schloss sie ihre Promotion am Institut für Arbeitsökonomik der Leibniz Universität Hannover ab. Seit März 2010 ist sie bei der Deutschen Bundesbank in der volkswirtschaftlichen Abteilung tätig. 